\title{
Adaptive Trajectory Tracking Control for Rotorcraft Using Incremental Backstepping Sliding Mode Control Strategy
}

\author{
Moon Gyeang Cho $\mathbb{D}$, Useok Jung, Jun-Young An, Yoo-Seung Choi, and Chang-Joo Kim \\ Department of Aerospace and Information Engineering, Konkuk University, Seoul 05029, Republic of Korea \\ Correspondence should be addressed to Chang-Joo Kim; cjkim@konkuk.ac.kr
}

Received 1 June 2021; Accepted 30 June 2021; Published 14 July 2021

Academic Editor: Xingling Shao

Copyright (c) 2021 Moon Gyeang Cho et al. This is an open access article distributed under the Creative Commons Attribution License, which permits unrestricted use, distribution, and reproduction in any medium, provided the original work is properly cited.

\begin{abstract}
This paper investigates the adaptive incremental backstepping sliding mode control for the rotorcraft trajectory-tracking control problem to enhance the robustness to the matched uncertainty in the model. First, the incremental dynamics is used for the control design to exclude the adverse effect of the mismatched model uncertainties on the trajectory-tracking performance. Secondly, the sliding-mode control strategy is adopted in the second design stage of the backstepping controller, and the effect of switching gains on the controller robustness is thoroughly studied using the rotorcraft model with different levels of the matched uncertainties. To clarify the robustness enhancement using the adaptive selection of switching gains, this paper chooses three different control structures consisting of the traditional backstepping control and two backstepping sliding mode controls with the fixed or adaptively adjusted switching gains. These control designs are applied to the trajectory-tracking control for the helical-turn maneuver of the Bo-105 helicopter to compare their relative robustness to the matched uncertainties. The results prove that adaptive incremental backstepping sliding mode control shows much higher robustness than other two designs, and the controller even with the fixed switching gains can be used to improve the robustness of the pure backstepping control design. Therefore, the present adaptive incremental backstepping sliding mode control is effectively applicable with the rotorcraft model which typically contains many different sources of both matched and mismatched uncertainties.
\end{abstract}

\section{Introduction}

Many of the aircraft's roles in the search and rescue, patrol, reconnaissance, and transport missions can be effectively performed by the rotorcraft with its unique vertical take-off and landing capabilities. These rotorcraft missions are typically highlighted by the wide operating range from hover to high forward speed, dangerous near-earth operations, and poor handling qualities over most of the operating flight envelop (OFE) of the rotorcraft $[1,2]$. Also, the flight safety and reliability highly depend on the uncertain and complex operating environments. In addition, the dynamic models commonly used for the flight control design typically suffer from serious model deficiency related to the complex rotor motions and simplified inflow dynamics $[1,3]$. Therefore, the control robustness to the external disturbances and unmodeled dynamics is extremely important for the mission success in the flight control system (FCS) design. In the last two decades, many nonlinear control designs rather than the linear feedback control theory have been developed and successfully implemented to enhance the mentioned robustness [4-10]. Among them, it is well known that the backstepping control (BSC) and sliding mode control (SMC) designs can provide a promising control solution when the incremental dynamics is used as a plant model and various adaptive control elements are adopted in their designs.

The incremental dynamics provides various advantages in control design over a full nonlinear rotorcraft dynamical model. First, it is inherently affine to the control. Therefore, BSC and SMC designs are straightforwardly applicable without resorting to complex affine transformation as shown in Refs. [11-14]. Secondly, the modeling errors due to external disturbances and mismatched uncertainties in the dynamics can be effectively cancelled out by the measured or predicted accelerations which are used in the control design [15-17]. Finally, BSC and SMC designs with the incremental dynamics 
provides the integral control function, which can further reduce the steady-state errors and additionally improve the robustness to external disturbances. It is well known that the incremental BSC (IBSC) design is not effective to compensate the matched disturbance, and its adaptive version typically requires a prior knowledge on the size of uncertainty or the uncertainty prediction [18]. Thus, the uncertainty compensation method such as the tuning function (TF), immersion and invariance (I\&I), and least-squares (LS) methods has been widely studied to effectively handle matched disturbances [19-23].

Each of these methods has different pros and cons. First, the LS-based control design is an indirect adaptive method which uses the recursively estimated disturbances or unknown model parameters to adaptively restructure the controller. For its applications, the persistent excitation condition must be held in order to acquire exact values of the unknown disturbances or parameters $[19,20]$. Furthermore, the stability proof for the closed-loop system with the LS-based method is extremely difficult when the controller is independently designed based on the certainty equivalence principle. On the other hand, TF- and I\&I-based methods typically use the Lyapunov-based design. Their integrated design of both the controller and observer can guarantee the stability of the corresponding closed-loop system. However, the TF-based method is known to provide inaccurate estimates for the unknown parameter or disturbance, whereas the I\&I-based method can provide an accurate estimation of the system uncertainties by adopting an additional shaping function. However, I\&I also may suffer from extreme design complexity because the shaping function should be determined with the solution of the associated partial differential equation. Even with different characteristics, above three methods have been successfully used in various adaptive IBSC applications to compensate the adverse effects of system uncertainties.

It is well known that incremental SMC (ISMC) combined with the above mentioned adaptive element is relatively more robust to the matched disturbances than IBSC when the size of the associated uncertainty is specified [14, 24, 25]. As an alternative, some of the adaptive ISMC design adopted the variable switching gain, the main purpose of which intends to increase the switching gain according to the prescribed size of the uncertainty [26-29]. There may exist many drawbacks of this approach. First, the switching gain determined considering the trajectory-tracking error may become ever increasing and finally destabilize the close-loop system as shown in Ref. [26-28]. In addition, the increased switching gain possibly causes the severe chattering phenomena and computes the control input over its saturation limit. Secondly, the prior knowledge on the size of uncertainty over OFE is impractical in real applications. After predicting the uncertainty, the switching gain can be adaptively adjusted to stabilize the close-loop system. However, even in this case, the unreasonable increase in the switching gain is typically unavoidable.

This paper mainly focuses on the incremental adaptive backstepping sliding mode control (AIBS-SMC) to enhance the controller robustness to the match uncertainties in the rotorcraft dynamics. First, the incremental rotorcraft dynamics are derived from the high fidelity rotorcraft model imple- mented in the HETLAS (Helicopter Trim, Linearization, and Simulation) program, which has been developed for the rotorcraft fly-by-wire flight control system program [30, 31]. Secondly, the slack variable approach [32-34] is adopted to obtain the corresponding square system, which can greatly reduce the workloads in the nonlinear control design. In addition, the unstable internal dynamics are stabilized using the concept of the stability augmentation system. Finally, the AIBS-SMC design is performed with the Lyapunovbased method to define the control structure and to establish the stability proof. To differentiate the control structures used in the paper, first, the AIBSC design is carried out using the TF-based method and followed by the detail design of the AIBS-SMC. The proposed methods are validated through a series of simulation works for the trajectory tracking control of the rotorcraft during the helical-turn maneuver defined in ADS-33E-PRF [2]. The level of the matched uncertainty in the rotorcraft model is adjusted up to $50 \%$ of the control effectiveness matrix of the nominal plant with a simple weight constant. And the robustness enhancement achievable with the present ABS-SMC is validated through the comparison of its results with those using the pure ABSC and ABS-SMC with the fixed switching gain.

\section{Incremental Dynamics}

The rotorcraft dynamics are represented by the following Euler equations and kinematical relations $[1,3]$.

$$
\begin{gathered}
\dot{\mathbf{v}}=\frac{1}{m} \mathbf{f}_{b}-\boldsymbol{\omega} \times \mathbf{v}, \\
\dot{\boldsymbol{\omega}}=\mathbf{J}^{-1}\left(\mathbf{m}_{b}-\boldsymbol{\omega} \times \mathbf{J} \boldsymbol{\omega}\right), \\
\boldsymbol{\omega}=\mathbf{L} \dot{\phi}, \\
\mathbf{v}=\mathbf{C} \dot{\mathbf{r}} .
\end{gathered}
$$

Here, the linear velocity $\mathbf{v}$, angular rate $\boldsymbol{\omega}$, Euler angles $\phi$, and the position $\mathbf{r}$ of the aircraft are represented by

$$
\mathbf{v}=\left(\begin{array}{c}
u \\
v \\
w
\end{array}\right), \boldsymbol{\omega}=\left(\begin{array}{l}
p \\
q \\
r
\end{array}\right), \boldsymbol{\phi}=\left(\begin{array}{l}
\phi \\
\theta \\
\psi
\end{array}\right), \mathbf{r}=\left(\begin{array}{l}
x \\
y \\
z
\end{array}\right) .
$$

And $m$ and $\mathbf{J}$ are the mass and moment of inertial matrix, respectively. The external forces and moments acting on the aircraft in the body-fixed frame are denoted by $\mathbf{f}_{b}$ and $\mathbf{m}_{b}$, which are typically derived using the component-based modeling techniques for the rotorcraft. As an example, $\mathbf{f}_{b}$ is typically represented by the sum of each contribution of the main rotor (mr), fuselage (fus), stabilizer (stb), tail rotor (tr), and gravity (grv) forces as

$$
\mathbf{f}_{b}=\mathbf{f}_{\mathrm{mr}}\left(\delta_{0}, \delta_{1 C}, \delta_{1 S}\right)+\mathbf{f}_{\mathrm{fus}}+\mathbf{f}_{\mathrm{stb}}+\mathbf{f}_{\mathrm{tr}}\left(\delta_{\mathrm{TR}}\right)+\mathbf{f}_{\mathrm{grv}} .
$$

For the control of the conventional helicopter, the pitch angles of the main and tail rotors are typically used. In such a case, the primary controls $\mathbf{u}_{p}=\left(\delta_{0}, \delta_{1 C}, \delta_{1 S}, \delta_{\mathrm{TR}}\right)^{T}$ consist 
of the collective, lateral cyclic, longitudinal cyclic pitches of the main rotor and collective pitch of the tail rotor, respectively. These controls cause the flap, lead-lag, and feathering motions of the rotor, which directly affect the forces $\left(\mathbf{f}_{\mathrm{mr}}, \mathbf{f}_{\mathrm{tr}}\right)$ and moments $\left(\mathbf{m}_{\mathrm{mr}}, \mathbf{m}_{\mathrm{tr}}\right)$ produced by the rotors. The associated dynamics (flap and lead-lag) and the resultant rotor forces and moment are typically derived for a high-fidelity math model by applying the blade element method (BEM) and by numerically integrating the nonlinear aerodynamic loads along the blade radial position [1, 3]. Furthermore, the inflow dynamics are modeled using the nonlinear air load distribution over the tip-path plane of the rotor. Therefore, the rotorcraft motion equations are generally the nonaffine system to the primary control $\mathbf{u}_{p}$ [35].

The system output for the trajectory-tracking control can be defined by the position and heading angle of the aircraft. The associated position and attitude dynamics are derived by applying the kinematics in Eq. (1) as [36, 37].

$$
\begin{gathered}
\ddot{\mathbf{r}}=\mathbf{C}^{-1}\left\{\frac{\mathbf{f}_{b}}{m}-(\mathbf{L} \dot{\boldsymbol{\phi}}) \times(\mathbf{C} \dot{\mathbf{r}})-\dot{\mathbf{C}} \dot{\mathbf{r}}\right\}, \\
\ddot{\boldsymbol{\phi}}=\mathbf{L}^{-1} \mathbf{J}^{-1}\left\{\mathbf{m}_{b}-(\mathbf{L} \dot{\boldsymbol{\phi}}) \times(\mathbf{J} \dot{\mathbf{\phi}})\right\}-\mathbf{L}^{-1} \dot{\mathbf{L}} \dot{\boldsymbol{\phi}} .
\end{gathered}
$$

Using the definition of the state vector $\mathbf{x}=(x, y, z, \phi, \theta, \psi)^{T}$ and assuming that there exists the system uncertainties $\mathbf{d}(\mathbf{x}, \dot{\mathbf{x}}$ , $\mathbf{u}_{p}$ ) due to the modeling error and external disturbances, the corresponding system dynamics for the trajectorytracking problem can be represented by adding the system uncertainties as

$$
\begin{gathered}
\ddot{\mathbf{x}}=\mathbf{f}\left(\mathbf{x}, \dot{\mathbf{x}}, \mathbf{u}_{p}\right)+\mathbf{d}\left(\mathbf{x}, \dot{\mathbf{x}}, \mathbf{u}_{p}\right), \\
\mathbf{y}=(x, y, z, \psi)^{T} .
\end{gathered}
$$

The dimension and relative degree of Eq. (5) are $n=12$ and $\rho_{r}=8$, respectively, because $\mathbf{u}_{p}$ first appears in the second time derivative of $\mathbf{y}$. And the four internal dynamics for $(\phi, \dot{\phi}, \theta, \dot{\theta})$ are unobservable from the output, and they are typically unstable over most of the rotorcraft's OFE. Therefore, it can be said that the rotorcraft nonlinear control may become extremely complex because of the nonaffine and underactuated nonminimum-phase system dynamics.

Two efficient strategies related to the nonaffine and nonminimum-phase system are adopted to relieve the design workloads. One is the incremental dynamic approach [4-8], and the other is the adoption of the slack variable method [32-34]. The first intends to simplify the system dynamics using the known or measured states at the previous time step. The motion equation in Eq. (5) can be written using the states $\left(\mathbf{x}_{0}, \dot{\mathbf{x}}_{0}\right)$ and control $\mathbf{u}_{p 0}$ at $t=t_{0}$ as

$$
\ddot{\mathbf{x}}_{0}=\mathbf{f}_{0}+\mathbf{d}_{0}=\mathbf{f}\left(\mathbf{x}_{0}, \dot{\mathbf{x}}_{0}, \mathbf{u}_{p 0}\right)+\mathbf{d}\left(\mathbf{x}_{0}, \dot{\mathbf{x}}_{0}, \mathbf{u}_{p 0}\right) .
$$

Using the incremental state and control and the Jacobian matrices shown in Eq. (7), the dynamics at the next time step $t=t_{0}+\Delta t$ can be approximated using the first order Taylor- series expansion as shown in Eq. (8).

$$
\begin{gathered}
\mathbf{x}=\mathbf{x}_{0}+\Delta \mathbf{x}, \\
\dot{\mathbf{x}}=\dot{\mathbf{x}}_{0}+\Delta \dot{\mathbf{x}}, \\
\mathbf{u}_{p}=\mathbf{u}_{p 0}+\Delta \mathbf{u}_{p}, \\
\mathbf{F}_{0}=\frac{\partial \mathbf{f}_{0}}{\partial(\mathbf{x}, \dot{\mathbf{x}})}, \mathbf{G}_{0}=\frac{\partial \mathbf{f}_{0}}{\partial \mathbf{u}_{p}}, \\
\mathbf{D}_{0}=\frac{\partial \mathbf{d}_{0}}{\partial(\mathbf{x}, \dot{\mathbf{x}})}, \mathbf{H}_{0}=\frac{\partial \mathbf{d}_{0}}{\partial \mathbf{u}_{p}}, \\
\ddot{\mathbf{x}}=\mathbf{f}\left(\mathbf{x}, \dot{\mathbf{x}}, \mathbf{u}_{p}\right), \\
\underbrace{\mathbf{f}_{0}+\mathbf{d}_{0}}_{\mathbf{x}_{0}}+\mathbf{F}_{0}\left(\begin{array}{c}
\Delta \mathbf{x} \\
\Delta \dot{\mathbf{x}}
\end{array}\right)+\mathbf{G}_{0} \Delta \mathbf{u}_{p}+\mathbf{D}_{0}\left(\begin{array}{c}
\Delta \mathbf{x} \\
\Delta \dot{\mathbf{x}}
\end{array}\right)+\mathbf{H}_{0} \Delta \mathbf{u}_{p} .
\end{gathered}
$$

$\Delta \mathbf{x}$ and $\Delta \dot{\mathbf{x}}$ should be cancelled out to get an efficient equation for control. If both variables cannot be eliminated, $\mathbf{D}_{0}\left(\begin{array}{c}\Delta \mathbf{x} \\ \Delta \dot{\mathbf{x}}\end{array}\right)$ is reflected in the state-related uncertainty, which can cause instability in the controller [29]. Therefore, staterelated uncertainty should be cancelled out by assuming that $\Delta \mathbf{x}$ and $\Delta \dot{\mathbf{x}}$ are negligibly small over the relatively small $\Delta t$. The following incremental dynamics is obtained.

$$
\ddot{\mathbf{x}}=\ddot{\mathbf{x}}_{0}+\mathbf{G}_{0} \Delta \mathbf{u}_{p}+\Delta \mathbf{d} \quad \text { where } \Delta \mathbf{d}=\mathbf{H}_{0} \Delta \mathbf{u}_{p} .
$$

Above approximated dynamics are inherently affine to the incremental control $\Delta \mathbf{u}_{p}$ and can be directly used for the nonlinear control design once the accelerations $\ddot{\mathbf{x}}_{0}$ are available from their measurement or prediction. More interestingly, the scheduling of $\mathbf{G}_{0}$ as in the gain-schedule concept for the linear control theory may allow an efficient implementation of the nonlinear control laws without installing the complex nonlinear rotorcraft model into the flight control computer.

The slack variable method has been developed to transform an underactuated dynamics into the corresponding fully actuated form by adding the required number of slack variables $\mathbf{u}_{s}$ [32-34]. The resultant dynamics by adopting this method can be written as

$$
\begin{gathered}
\ddot{\mathbf{x}}=\ddot{\mathbf{x}}_{0}+\mathbf{G} \Delta \mathbf{u}+\boldsymbol{\xi}+\Delta \mathbf{d}, \\
\mathbf{y}=\mathbf{x}=(x, y, z, \phi, \theta, \psi)^{T},
\end{gathered}
$$

where

$$
\begin{array}{cc}
\mathbf{G} & =\left(\mathbf{G}_{0}, \mathbf{G}_{s}\right) \quad \Delta \mathbf{u}=\left(\Delta \mathbf{u}_{p}^{T}, \Delta \mathbf{u}_{s}^{T}\right)^{T}, \\
\boldsymbol{\xi} & =-\mathbf{G}_{s} \Delta \mathbf{u}_{s} \quad \mathbf{u}=\mathbf{u}_{0}+\Delta \mathbf{u},
\end{array}
$$

and $\Delta \mathbf{u}_{s}=\left(\Delta u_{5}, \Delta u_{6}\right)$ is a slack variable that does not respond to dynamic equation. If the matrix $\mathbf{G}_{s}$ is set as 
$\mathbf{G}_{s}=\left[\begin{array}{llllll}0 & 0 & 0 & 1 & 0 & 0 \\ 0 & 0 & 0 & 0 & 1 & 0\end{array}\right]^{T}, \quad \boldsymbol{\xi} \quad$ is defined as $\boldsymbol{\xi}=$ $\left[\begin{array}{llllll}0 & 0 & 0 & \Delta u_{5} & \Delta u_{6} & 0\end{array}\right]$. Though the slack-variable method, $\mathbf{G}$, can find the inverse matrix. The matrix $\mathbf{G}_{s}$ is typically prescribed to get the square $\mathbf{G}$ with a good condition number. The attitude angles are included in the system output, and the resultant system dynamics of Eq. (10) are fully actuated. At this moment, it is worth mentioning that all trajectory for six output states cannot be accurately prescribed because their independent controls are impossible only with the four primary controls of the rotorcraft. This issue will be addressed in the application section.

An accurate prediction of the acceleration $\ddot{\mathbf{x}}_{0}$ is required to use the incremental dynamics for the control design. The aircraft navigation systems typically provide the linear accelerations, attitude angles, and body-angular rates except the angular acceleration. Various algorithms have been developed to accurately predict the angular acceleration. Ekeren [16] adopted the wash-out filter to predict the angular acceleration using the measured angular rates. Similarly, Sieberling [15] used the linear predictive filter, the coefficients of which have been trained off-line using the data generated with a reference math model and applying the least-square method. Difficulties in the stability proof for the overall closed-loop system and the dependency on the math model become common drawbacks of these filtered approaches. Applications of the backward difference formulas (BDF) [17] appear promising in that they are extremely simple, independent of a specific model and incur no issues on the stability proof. These BDF methods use the measured angular velocities, and the following two- and five-point BDFs have been widely adopted for the prediction of the body angular acceleration $\dot{\omega}_{k}$ at $t=t_{k}$ in the literatures $[16,38]$

$$
\begin{gathered}
\hat{\dot{\omega}}_{k}=\frac{1}{\Delta t}\left(\omega_{k}-\omega_{k-1}\right), \\
\widehat{\dot{\omega}}_{k}=\frac{1}{12 \Delta t}\left(25 \omega_{k}-48 \omega_{k-1}+36 \omega_{k-2}-16 \omega_{k-3}+3 \omega_{k-4}\right),
\end{gathered}
$$

where $\omega_{j},(j=k, k-1, \cdots)$ denotes the measured body angular rate and $\widehat{\dot{\omega}}_{k}$ is the estimated angular acceleration. For the comparison of the prediction accuracies achievable with each BDF, the roll responses of the Bo-105 helicopter to the doublet lateral cyclic input are simulated from the trimmed level forward flight at 60 knots, and the angular predicted acceleration with $\Delta t=0.01$ seconds is compared in Figure 1. The result shows that the two-point BDF provides an accurate nonoscillatory prediction, whereas five-point one highly suffers from the Runge phenomena around the time of a sharp change in the angular rate. Thus, the two-point BDF shown in Eq. (12) is adopted throughout this paper.

\section{Design of Incremental Backstepping Sliding Mode Control}

3.1. Adaptive Incremental Backstepping Control Design. First, the design of the adaptive IBSC with the tuning function is addressed in this section. An integrated design approach is adopted rather than a recursive one used in the standard IBSC design to simplify the derivation. Prescribing the desired position trajectory $\mathbf{x}_{d}$, the corresponding position error $\mathbf{z}_{1}$ is defined by

$$
\mathbf{z}_{1}=\mathbf{x}-\mathbf{x}_{d}
$$

As in the BSC design $[39,40]$, the velocity error $\mathbf{z}_{2}$ is typically defined with the pseudocontrol $\boldsymbol{\alpha}$, which differs from the desired velocity $\dot{\mathbf{x}}_{d}$ in that it will be designed to stabilize the closed-loop system.

$$
\mathbf{z}_{2}=\dot{\mathbf{x}}-\boldsymbol{\alpha}
$$

The dynamics for new states $\left(\mathbf{z}_{1}, \mathbf{z}_{2}\right)$ are derived by differentiating Eqs. (14) and (15) and using Eq. (10).

$$
\begin{gathered}
\dot{\mathbf{z}}_{1}=\mathbf{z}_{2}+\boldsymbol{\alpha}-\dot{\mathbf{x}}_{d}, \\
\dot{\mathbf{z}}_{2}=\ddot{\mathbf{x}}_{0}+\mathbf{G} \Delta \mathbf{u}+\boldsymbol{\xi}+\Delta \mathbf{d}-\dot{\boldsymbol{\alpha}} .
\end{gathered}
$$

With the commonly used assumption of the slow variations of both $\boldsymbol{\xi}$ and $\Delta \mathbf{d}(\dot{\boldsymbol{\xi}} \approx 0, \Delta \dot{\mathbf{d}} \approx 0)$, the dynamics of their predictions $(\widehat{\boldsymbol{\xi}}, \Delta \widehat{\mathbf{d}})$ can be related to those for the prediction errors $(\tilde{\xi}, \Delta \tilde{\mathbf{d}})$ as

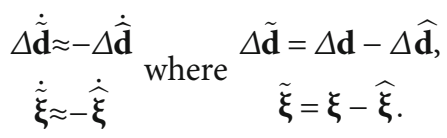

Using the control Lyapunov function (CLF) $V$ defined in Eq. (18) with the suitably chosen positive-definite weight matrices $\left(\mathbf{Q}, \Gamma_{\mathbf{d}}, \Gamma_{\xi}\right)$, the controller structure can be designed to meet the stability condition $\dot{V} \leq 0$ as shown in Eq. (19).

$$
\begin{gathered}
V=\frac{1}{2} \mathbf{z}_{1}^{T} \mathbf{Q}^{-1} \mathbf{z}_{1}+\frac{1}{2} \mathbf{z}_{2}^{T} \mathbf{z}_{2}+\frac{1}{2} \tilde{\xi}^{T} \Gamma_{\xi}^{-1} \tilde{\xi}+\frac{1}{2} \Delta \tilde{\mathbf{d}}^{T} \Gamma_{\mathbf{d}}^{-1} \Delta \widetilde{\mathbf{d}}, \\
\dot{V}=\mathbf{z}_{1}^{T} \mathbf{Q}^{-1} \dot{\mathbf{z}}_{1}+\mathbf{z}_{2}^{T} \dot{\mathbf{z}}_{2}+\tilde{\xi}^{T} \Gamma_{\xi}^{-1} \dot{\tilde{\xi}}+\Delta \tilde{\mathbf{d}}^{T} \Gamma_{\mathbf{d}}^{-1} \Delta \dot{\tilde{\mathbf{d}}} .
\end{gathered}
$$

Substituting Eq. (16) into Eq. (19), the time derivative of CLF is reduced to

$$
\begin{gathered}
\dot{V}=\mathbf{z}_{1}^{T} \mathbf{Q}^{-1}\left(\boldsymbol{\alpha}-\dot{\mathbf{x}}_{d}\right)+\mathbf{z}_{2}^{T}\left(\mathbf{Q}^{-T} \mathbf{z}_{1}+\ddot{\mathbf{x}}_{0}+\mathbf{G} \Delta \mathbf{u}+\boldsymbol{\xi}+\Delta \mathbf{d}-\dot{\boldsymbol{\alpha}}\right) \\
+\tilde{\boldsymbol{\xi}}^{T} \Gamma_{\xi}^{-1} \dot{\tilde{\xi}}+\Delta \tilde{\mathbf{d}}^{T} \Gamma_{\mathbf{d}}^{-1} \Delta \dot{\tilde{\mathbf{d}}} .
\end{gathered}
$$

Replacing $\xi$ and $\Delta \mathbf{d}$ with their estimates in Eq. (17), Eq. (20) becomes 

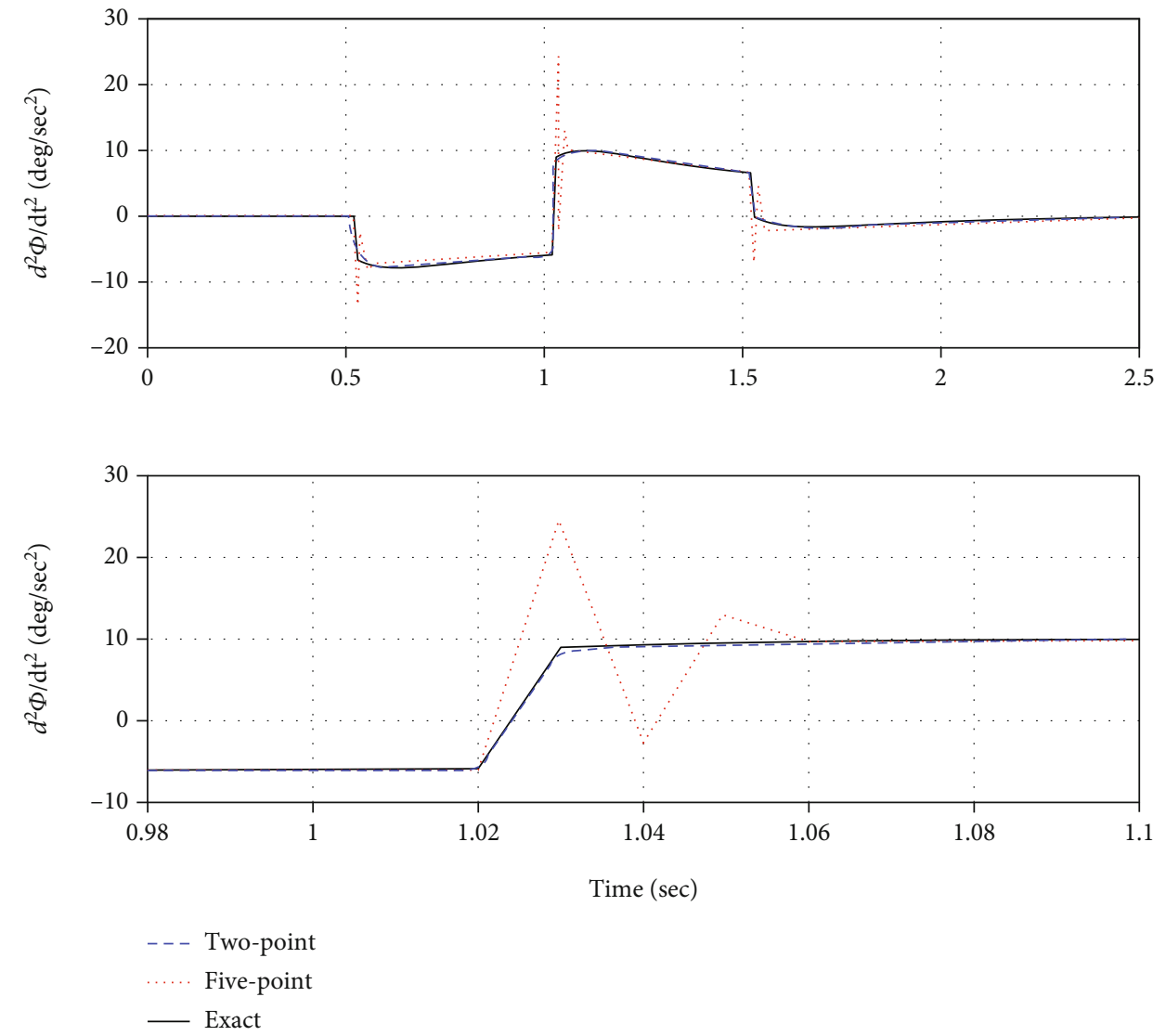

FIGURE 1: Comparison of the angular acceleration predicted with backward difference formulas.

$$
\begin{gathered}
\dot{V}=\mathbf{z}_{1}^{T} \mathbf{Q}^{-1}\left(\boldsymbol{\alpha}-\dot{\mathbf{x}}_{d}\right)+\mathbf{z}_{2}^{T}\left(\mathbf{Q}^{-T} \mathbf{z}_{1}+\ddot{\mathbf{x}}_{0}+\mathbf{G} \Delta \mathbf{u}+\widehat{\boldsymbol{\xi}}+\Delta \widehat{\mathbf{d}}-\dot{\boldsymbol{\alpha}}\right) \\
+\tilde{\xi}^{T}\left(\mathbf{z}_{2}-\Gamma_{\xi}^{-1} \dot{\widehat{\xi}}\right)+\Delta \tilde{\mathbf{d}}^{T}\left(\mathbf{z}_{2}-\Gamma_{\mathbf{d}}^{-1} \Delta \dot{\widehat{\mathbf{d}}}\right)
\end{gathered}
$$

Therefore, the controller structure and the update formulas for the slack variables and the disturbance can be derived by selecting the gain matrices of $\mathbf{K}_{1}>0$ and $\mathbf{K}_{2}>0$ to meet the stability condition as shown in Eq. (24).

$$
\begin{gathered}
\mathbf{K}_{1} \mathbf{z}_{1}=-\mathbf{Q}^{-1}\left(\boldsymbol{\alpha}-\dot{\mathbf{x}}_{d}\right), \\
\mathbf{K}_{2} \mathbf{z}_{2}=-\left(\mathbf{Q}^{-T} \mathbf{z}_{1}+\ddot{\mathbf{x}}_{0}+\mathbf{G} \Delta \mathbf{u}+\widehat{\boldsymbol{\xi}}+\Delta \widehat{\mathbf{d}}-\dot{\boldsymbol{\alpha}}\right), \\
\Delta \dot{\widehat{\mathbf{d}}}=\Gamma_{\mathbf{d}} \mathbf{z}_{2}, \\
\dot{\widehat{\xi}}=\Gamma_{\xi} \mathbf{z}_{2}, \\
\Delta \mathbf{u}=-\mathbf{G}^{-1}\left(\mathbf{K}_{2} \mathbf{z}_{2}+\mathbf{Q}^{-T} \mathbf{z}_{1}+\ddot{\mathbf{x}}_{0}+\widehat{\xi}+\Delta \widehat{\mathbf{d}}-\dot{\boldsymbol{\alpha}}\right), \\
\dot{V}=-\mathbf{z}_{1}^{T} \mathbf{K}_{1} \mathbf{z}_{1}-\mathbf{z}_{2}^{T} \mathbf{K}_{2} \mathbf{z}_{2} \leq 0 .
\end{gathered}
$$

In a case when the disturbance is ignored in the controller design, $\Delta \mathbf{u}$ in Eq. (23) and $\dot{V}$ in Eq. (24) are reduced to

$$
\begin{gathered}
\Delta \mathbf{u}=-\mathbf{G}^{-1}\left(\mathbf{K}_{2} \mathbf{z}_{2}+\mathbf{Q}^{-T} \mathbf{z}_{1}+\ddot{\mathbf{x}}_{0}+\widehat{\boldsymbol{\xi}}-\dot{\boldsymbol{\alpha}}\right), \\
\dot{V}=-\mathbf{z}_{1}^{T} \mathbf{K}_{1} \mathbf{z}_{1}-\mathbf{z}_{2}^{T} \mathbf{K}_{2} \mathbf{z}_{2}+\mathbf{z}_{2}^{T} \Delta \mathbf{d} .
\end{gathered}
$$

The closed-loop system is stabilizable when the follow condition is satisfied by

$$
-\mathbf{z}_{1}^{T} \mathbf{K}_{1} \mathbf{z}_{1}-\mathbf{z}_{2}^{T} \mathbf{K}_{2} \mathbf{z}_{2}+\mathbf{z}_{2}^{T} \Delta \mathbf{d}<0
$$

Therefore, it can be claimed that the feedback control with the predicted disturbance as shown in Eq. (23) plays a crucial role in stabilizing the closed-loop system even though the disturbance update formula commonly shows poor accuracy. On the other hand, the predicted disturbance may unreasonably grow when there exists a large deviation in the velocity trajectory as shown Eq. (22). Even in this case, the closed-loop system is stabilizable whenever the aircraft control with the disturbance feedback has enough margin for all channels.

3.2. Adaptive Incremental Backstepping Sliding Mode Control Design. The adaptive incremental backstepping sliding-mode control (AIBS-SMC) design uses the sliding variable $\mathbf{s}$ and associated switching control [28, 41, 42]. In the standard SMC design, the position error $\mathbf{e}=\mathbf{x}-\mathbf{x}_{d}$ and its time derivative $\dot{\mathbf{e}}$ are combined to define the sliding surface $\mathbf{s}$, whereas the velocity error $\mathbf{z}_{2}$ designed for the BSC design is typically 
adopted for the BS-SMC control rather than $\dot{\mathbf{e}}$ as shown in the following definition.

$$
\mathbf{s}=\mathbf{z}_{2}+\mathbf{C} \mathbf{z}_{1}, \quad(\mathbf{C}>0) .
$$

In this paper, the CLF $W$ shown in Eq. (28) is used to define the control structure.

$$
W=\frac{1}{2} \mathbf{z}_{1}^{T} \mathbf{Q}^{-1} \mathbf{z}_{1}+\frac{1}{2} \mathbf{s}^{T} \mathbf{s}+\frac{1}{2} \tilde{\xi}^{T} \Gamma_{\xi}^{-1} \tilde{\xi}+\frac{1}{2} \Delta \tilde{\mathbf{d}}^{T} \Gamma_{\mathbf{d}}^{-1} \Delta \tilde{\mathbf{d}} .
$$

The time derivatives of $\dot{\mathbf{s}}$ and $\dot{W}$ are derived as

$$
\begin{gathered}
\dot{\mathbf{s}}=\dot{\mathbf{z}}_{2}+\mathbf{C} \dot{\mathbf{z}}_{1}=\ddot{\mathbf{x}}_{0}+\mathbf{G} \Delta \mathbf{u}+\boldsymbol{\xi}+\Delta \mathbf{d}-\dot{\boldsymbol{\alpha}}+\mathbf{C} \dot{\mathbf{z}}_{1}, \\
\dot{W}=\mathbf{z}_{1}^{T} \mathbf{Q}^{-1} \dot{\mathbf{z}}_{1}+\mathbf{s}^{T} \dot{\mathbf{s}}+\tilde{\boldsymbol{\xi}}^{T} \Gamma_{\xi}^{-1} \dot{\tilde{\xi}}+\Delta \tilde{\mathbf{d}}^{T} \Gamma_{\mathbf{d}}^{-1} \Delta \dot{\tilde{\mathbf{d}}} .
\end{gathered}
$$

$\dot{W}$ is derived using the similar process used for Eq. (21), and the result is represented by

$$
\begin{aligned}
\dot{W}=\mathbf{z}_{1}^{T} \mathbf{Q}^{-1}\left(\boldsymbol{\alpha}-\dot{\mathbf{x}}_{d}-\mathbf{C} \mathbf{z}_{1}\right)+\mathbf{s}^{T}\left(\ddot{\mathbf{x}}_{0}+\mathbf{G} \Delta \mathbf{u}+\widehat{\boldsymbol{\xi}}+\Delta \widehat{\mathbf{d}}-\dot{\boldsymbol{\alpha}}+\mathbf{C} \dot{\mathbf{z}}_{1}+\mathbf{Q}^{-T} \mathbf{z}_{1}\right), \\
+\tilde{\xi}^{T}\left(\Gamma_{\xi}^{-1} \dot{\tilde{\xi}}+\mathbf{s}\right)+\Delta \tilde{\mathbf{d}}^{T} \Gamma_{\mathbf{d}}^{-1}(\Delta \dot{\tilde{\mathbf{d}}}+\mathbf{s}) .
\end{aligned}
$$

Therefore, the control and update formulas can be constructed as

$$
\begin{gathered}
\mathbf{K}_{1} \mathbf{z}_{1}=-\mathbf{Q}^{-1}\left(\boldsymbol{\alpha}-\dot{\mathbf{x}}_{d}-\mathbf{C} \mathbf{z}_{1}\right), \\
\mathbf{K}_{s} \mathbf{s}=-\left(\ddot{\mathbf{x}}_{0}+\mathbf{G} \Delta \mathbf{u}_{e q}+\widehat{\xi}+\Delta \widehat{\mathbf{d}}-\dot{\boldsymbol{\alpha}}+\mathbf{C} \dot{\mathbf{z}}_{1}+\mathbf{Q}^{-T} \mathbf{z}_{1}\right) \\
\Delta \dot{\widehat{\mathbf{d}}}=\Gamma_{\mathbf{d}} \mathbf{s}=\Gamma_{\mathbf{d}}\left(\mathbf{z}_{2}+\mathbf{C} \mathbf{z}_{1}\right) \\
\dot{\hat{\xi}}=\Gamma_{\xi} \mathbf{s}=\Gamma_{\xi}\left(\mathbf{z}_{2}+\mathbf{C} \mathbf{z}_{1}\right)
\end{gathered}
$$

Using the concept of the equivalent control as in the standard SMC [14], the incremental control can be split into the equivalent and switching ones with $\Delta \mathbf{u}=\Delta \mathbf{u}_{e q}+\Delta \mathbf{u}_{s w}$. Then, $\Delta \mathbf{u}_{e q}$ can be defined with reference to Eq. (31) as

$$
\Delta \mathbf{u}_{e q}=-\mathbf{G}^{-1}\left(\mathbf{K}_{s} \mathbf{s}+\mathbf{C} \dot{\mathbf{z}}_{1}+\mathbf{Q}^{-T} \mathbf{z}_{1}+\ddot{\mathbf{x}}_{0}+\widehat{\boldsymbol{\xi}}+\Delta \widehat{\mathbf{d}}-\dot{\boldsymbol{\alpha}}\right) .
$$
obtain

Substituting Eq. (31) and Eq. (32) into Eq. (30), we can

$$
\dot{W}=-\mathbf{z}_{1}^{T} \mathbf{K}_{1} \mathbf{z}_{1}-\mathbf{s}^{T} \mathbf{K}_{s} \mathbf{s}+\mathbf{s}^{T} \mathbf{G} \Delta \mathbf{u}_{\mathrm{sw}} .
$$

Thus, the switching control can be designed using the signum function $\operatorname{sgn}(\cdot)$ to satisfy the required stability condition with $\mathbf{K}_{s}>0$ and $\boldsymbol{\Lambda}_{s w}>0$ as

$$
\begin{gathered}
\Delta \mathbf{u}_{\mathrm{sw}}=-\mathbf{G}^{-1} \boldsymbol{\Lambda}_{\mathrm{sw}} \operatorname{sgn}(\mathbf{s}), \\
\dot{W}=-\mathbf{z}_{1}^{T} \mathbf{K}_{1} \mathbf{z}_{1}-\mathbf{s}^{T} \mathbf{K}_{\mathrm{s}} \mathbf{s}-\boldsymbol{\Lambda}_{\mathrm{sw}}\|\mathbf{s}\| \leq 0 .
\end{gathered}
$$

With $\mathbf{C}=0$, the controller structure for AIBS-SMC is the same as that of AIBSC except the switching control. However, this additional switching control can further improve the closed-loop stability as shown in Eq. (34) with $\mathbf{K}_{s}=\mathbf{K}_{2}$. In a case when the disturbance is ignored in the AIBS-SMC design, $\Delta \mathbf{u}_{e q}$ in Eq. (32) and $\dot{W}$ in Eq. (33) are reduced to

$$
\begin{gathered}
\Delta \mathbf{u}_{e q}=-\mathbf{G}^{-1}\left(\mathbf{K}_{s} \mathbf{s}+\mathbf{C} \dot{\mathbf{z}}_{1}+\mathbf{Q}^{-T} \mathbf{z}_{1}+\ddot{\mathbf{x}}_{0}+\widehat{\xi}-\dot{\mathbf{\alpha}}\right), \\
\dot{W}=-\mathbf{z}_{1}^{T} \mathbf{K}_{1} \mathbf{z}_{1}-\mathbf{s}^{T} \mathbf{K}_{s} \mathbf{s}-\boldsymbol{\Lambda}_{\mathrm{sW}}\|\mathbf{s}\|+\mathbf{s}^{T} \Delta \mathbf{d} .
\end{gathered}
$$

Thus, the stability of the closed-loop system can be guaranteed only when the follow condition is satisfied by

$$
-\boldsymbol{\Lambda}_{\mathrm{sw}}\|\mathbf{s}\|+\mathbf{s}^{T} \Delta \mathbf{d}<0 .
$$

There may exist three selectable options to meet the above condition. First, in a case when the upper bound of disturbance is known in prior, the switching gain $\boldsymbol{\Lambda}_{\mathrm{sw}}$ can be chosen to satisfy Eq. (36). This approach seems to be impractical since it is difficult to obtain a prior knowledge on the mentioned upper bound in real applications. As a second option, uncertainty is modeled using prediction method such as in Eq (17). This method does compensate for uncertainty not by using a variable switching gain, but by predicting uncertainty. Therefore, only the consideration on the parameter estimate $\Delta \widehat{\mathbf{d}}$ is required with the fixed switching gain. This method has the same form as that of the TFbased AIBSC $[14,24,25]$, but it might be impractical when the uncertainties are estimated with poor accuracy. This paper takes another option using a variable switching gain. This method is commonly used in ASMC [26-28] and does not require a prior knowledge on the size of uncertainty because the switching gain is typically adjusted using the update formula derived from the Lyapunov-based stability analysis. Since the size of uncertainty is typically unknown, the predicted switching gain $\widehat{\Lambda}_{\mathrm{sw}}$ rather than $\boldsymbol{\Lambda}_{\mathrm{sw}}$ must be used in the switching control. The required update formula can be derived using the prediction error of $\tilde{\Lambda}_{\mathrm{sw}}=\Lambda_{\mathrm{sw}}-$ $\dot{\Lambda}_{\text {sW }}$ and the following CLF $W$.

$$
W=\frac{1}{2} \mathbf{z}_{1}^{T} \mathbf{Q}^{-1} \mathbf{z}_{1}+\frac{1}{2} \mathbf{s}^{T} \mathbf{s}+\frac{1}{2} \tilde{\xi}^{T} \Gamma_{\xi}^{-1} \tilde{\xi}+\frac{1}{2} \tilde{\Lambda}_{\mathrm{sw}}^{T} \boldsymbol{\Gamma}_{\boldsymbol{\Lambda}}^{-1} \tilde{\Lambda}_{\mathrm{sw}}
$$

Assume that desired switching gains $\Lambda_{s w}$ meet the condition of Eq (36). The time derivative $\dot{W}$ is derived using the similar process used for Eq. (30). And by applying the $\Delta \mathbf{u}$ $=\Delta \mathbf{u}_{e q}+\Delta \mathbf{u}_{s w}$, the switch gain can be updated to meet the stability condition using

$$
\begin{gathered}
\dot{\hat{\boldsymbol{\Lambda}}}_{\mathrm{sw}}=\boldsymbol{\Gamma}_{\boldsymbol{\Lambda}}\|\mathbf{s}\|, \\
\Delta \mathbf{u}_{\mathrm{eq}}=-\mathbf{G}^{-1}\left(\mathbf{K}_{\mathrm{s}} \mathbf{s}+\mathbf{C} \dot{\mathbf{z}}_{1}+\mathbf{Q}^{-T} \mathbf{z}_{1}+\ddot{\mathbf{x}}_{0}+\widehat{\xi}-\dot{\mathbf{a}}\right), \\
\Delta \mathbf{u}_{\mathrm{sw}}=-\mathbf{G}^{-1} \widehat{\Lambda}_{\mathrm{sw}} \operatorname{sgn}(\mathbf{s}) .
\end{gathered}
$$



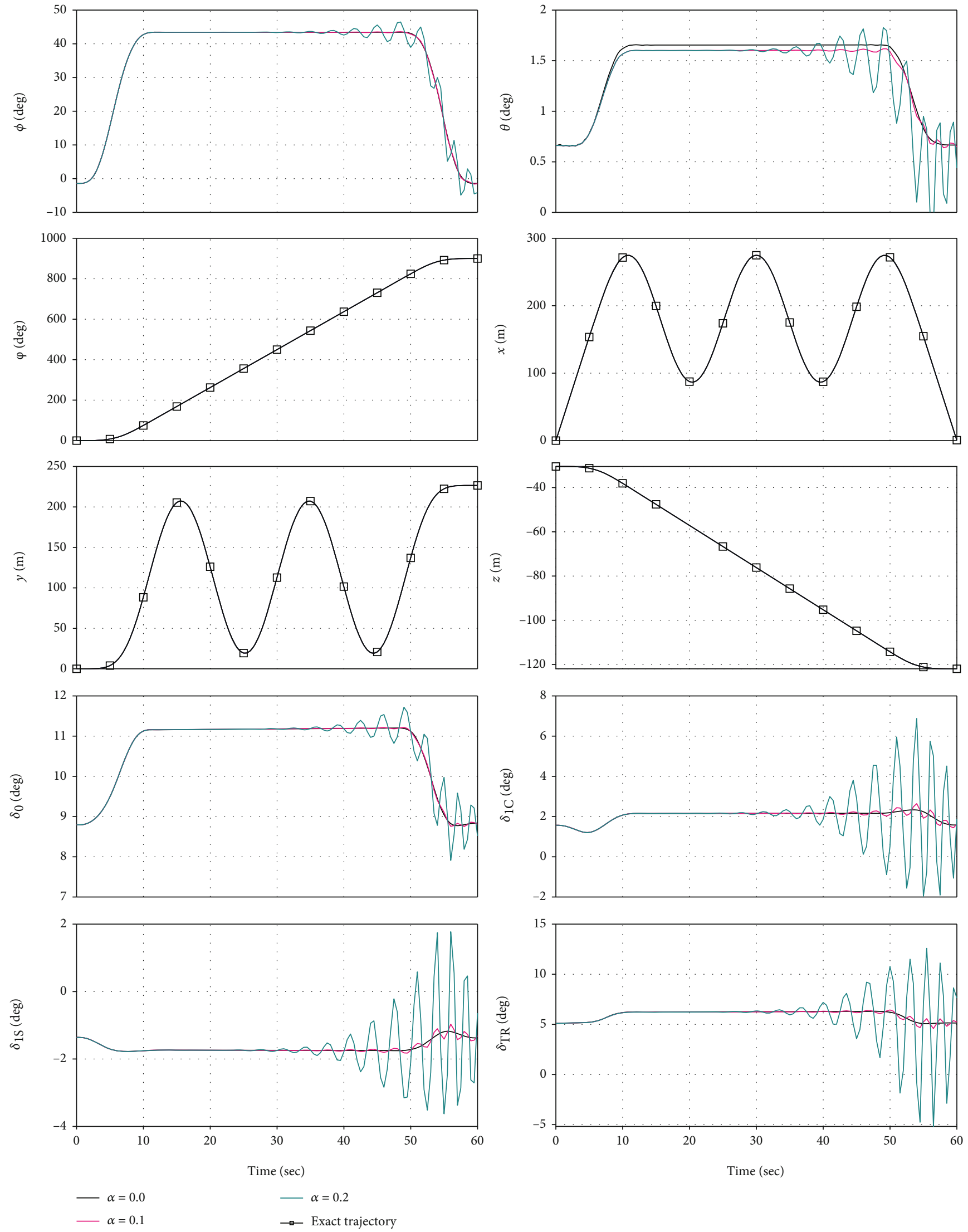

Figure 2: Simulated trajectories and feedback controls for the IBSC design with different levels of mismatched uncertainties. 

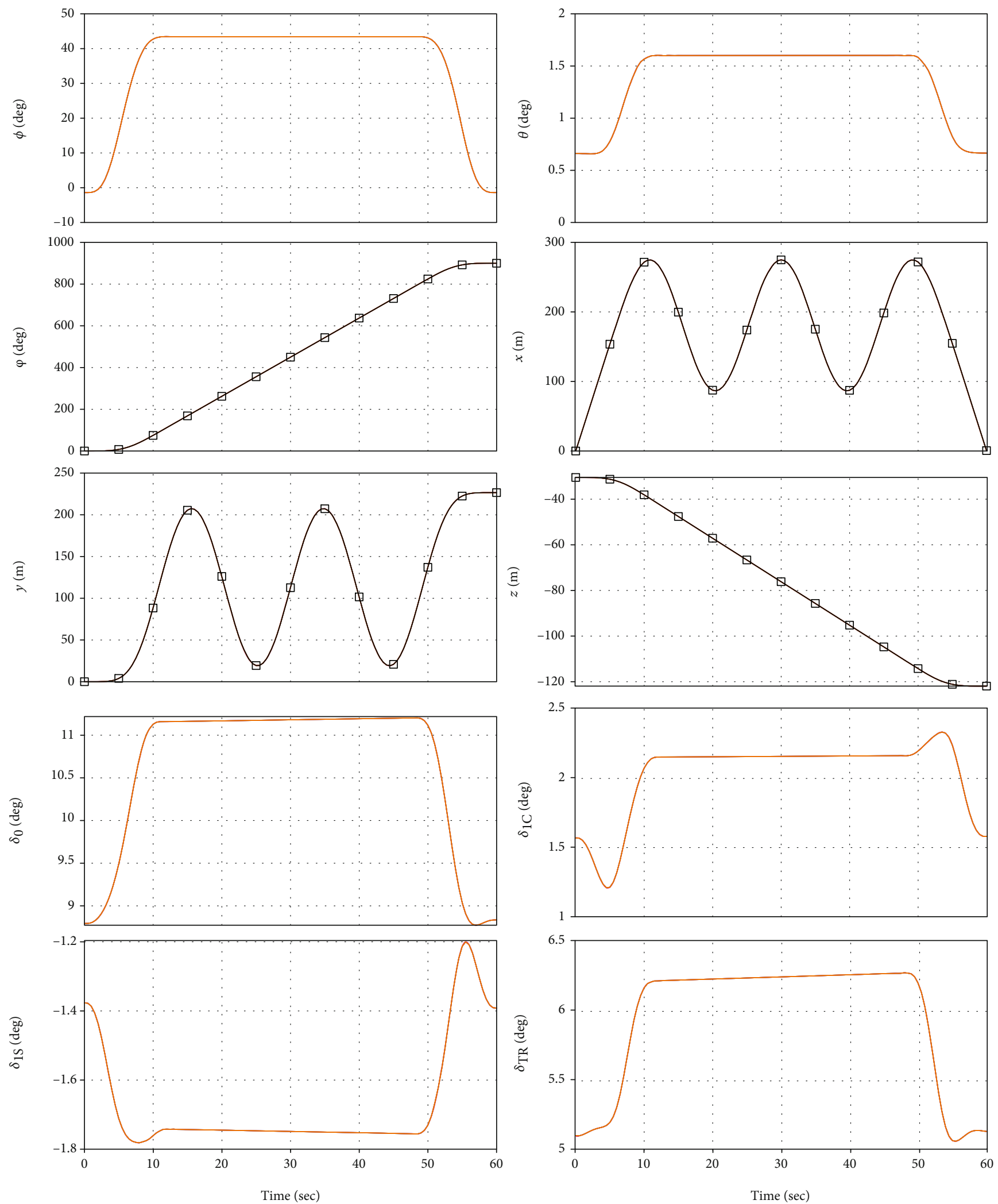

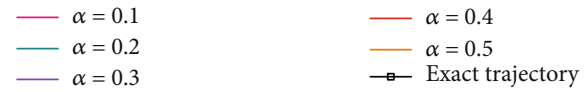

FIGURE 3: Simulated trajectories and feedback controls for the IBSC-SMC design with different levels of mismatched uncertainties with the fixed switching gain of $\Lambda_{\mathrm{sw}}=0.01$ 

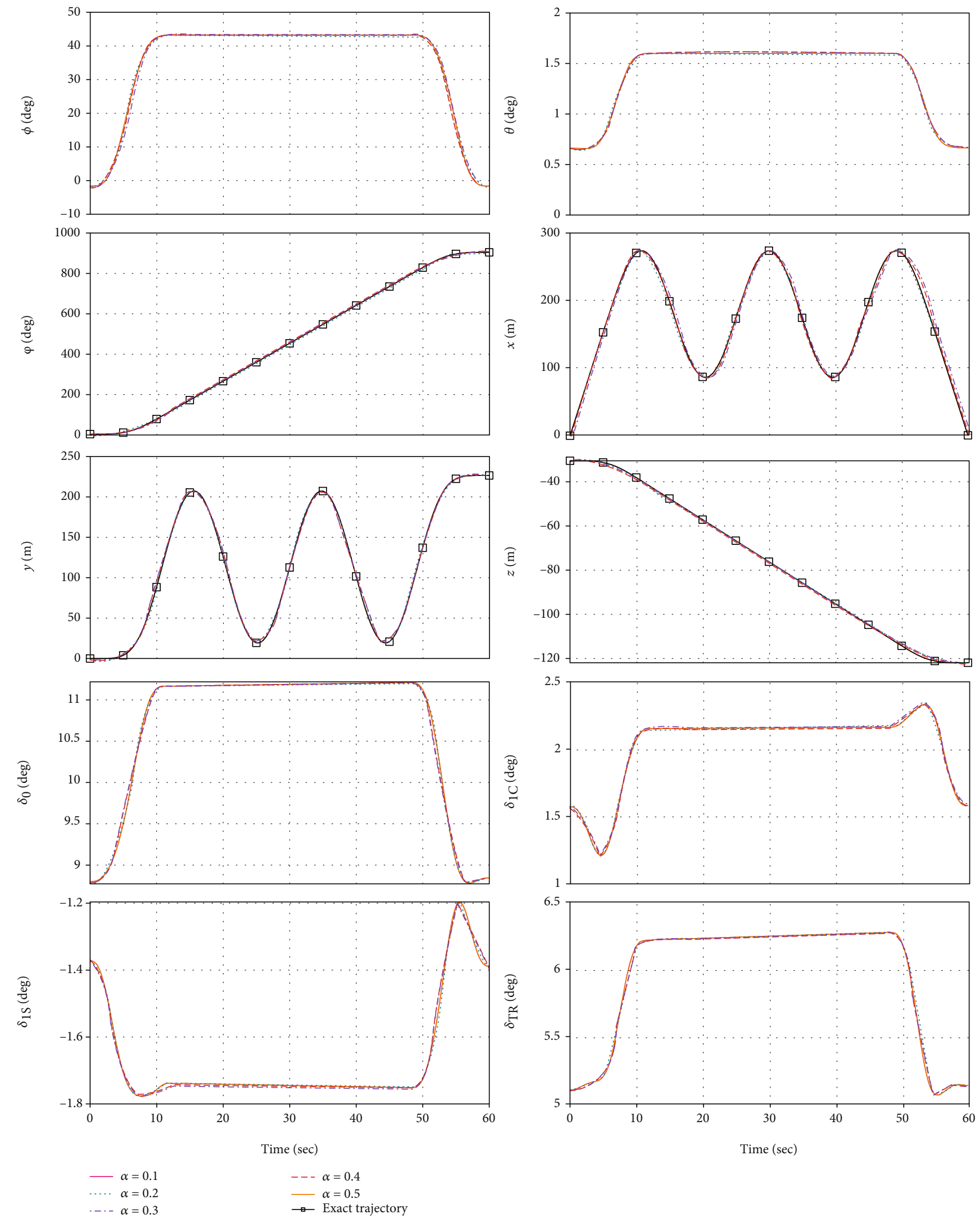

FIGURE 4: Simulated trajectories and feedback controls for the AIBSC-SMC design with different levels of mismatched uncertainties. 

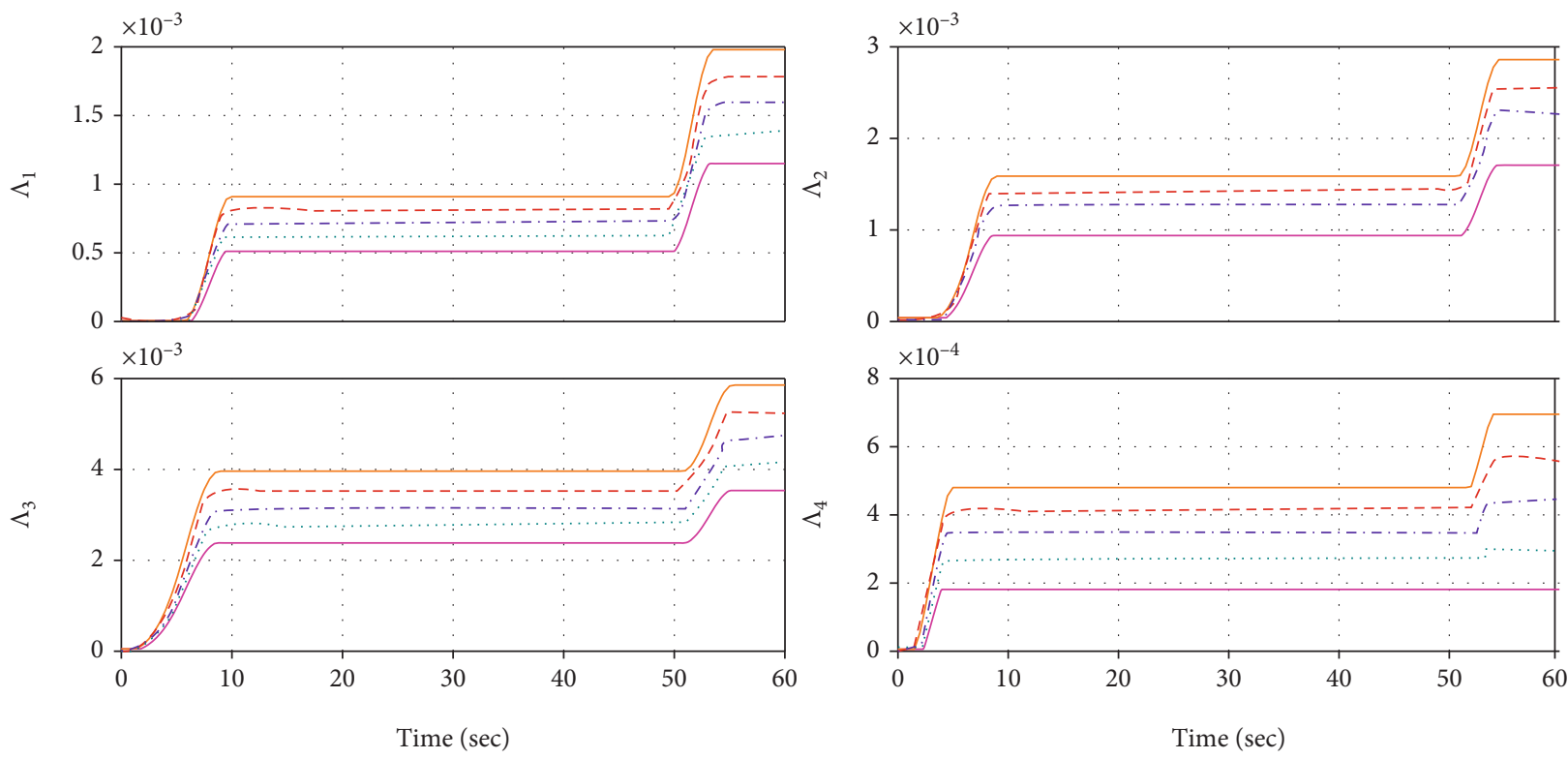

Figure 5: Variation of adaptive switching gains with different levels of mismatched uncertainties.

The stability condition results in

$$
\dot{W}=-\mathbf{z}_{1}^{T} \mathbf{K}_{1} \mathbf{z}_{1}-\mathbf{s}^{T} \mathbf{K}_{s} \mathbf{s}-\boldsymbol{\Lambda}_{\mathrm{sw}}\|\mathbf{s}\|+\mathbf{s}^{T} \Delta \mathbf{d}
$$

Under the assumption $\mathrm{Eq}$ (36), the following condition is always met.

$$
-\mathbf{z}_{1}^{T} \mathbf{K}_{1} \mathbf{z}_{1}-\mathbf{s}^{T} \mathbf{K}_{s} \mathbf{s}-\boldsymbol{\Lambda}_{\mathrm{sw}}\|\mathbf{s}\|+\mathbf{s}^{T} \Delta \mathbf{d}<0
$$

Therefore, AIBSC-SMC stabilizes the closed loop system. However, the adaptive law in Eq (38) leads to monotonically increasing switching gain due to switching control property. To eliminate switching gain drift problem, the dead zone technique used in ref [28] is adopted

$$
\dot{\Lambda}=\left\{\begin{array}{ll}
\dot{\Lambda}=\Gamma_{\Lambda}\|\mathbf{s}\| & \text { if }|\mathbf{s}|<\varepsilon \\
0 & \text { if }|\mathbf{s}| \geq \varepsilon
\end{array},\right.
$$

where $\varepsilon$ is the dead zone size. This method can cause steady-state error, but steady-state error can be reduced by setting the appropriate $\varepsilon$. In this paper, the size of $\varepsilon$ is selected using the experimental simulation result.

\section{Application and Discussion}

The robustness to the matched uncertainties, achievable with the proposed AIBS-SMC design, has been demonstrated through simulation studies along with the AIBSC design and the AIBS-SMC with the fixed switching gain. The matched uncertainties are reflected in the incremental dynamics, and their levels are defined by the sizing parameter $\alpha$ as

$$
\begin{aligned}
& \ddot{\mathbf{x}}=\ddot{\mathbf{x}}_{0}+\mathbf{G} \Delta \mathbf{u}+\boldsymbol{\xi}+\Delta \mathbf{d}, \\
& =\ddot{\mathbf{x}}_{0}+(1+\alpha) \mathbf{G} \Delta \mathbf{u}+\boldsymbol{\xi} .
\end{aligned}
$$

Therefore, $\alpha=0$ corresponds to the control design for the nominal dynamics. This paper validates the controller performance using the high-fidelity Bo-105 helicopter model implemented in the HETLAS $[30,31]$ The 4 th order Runge-Kutta integrator is adopted for the flight simulation with the timestep size of 0.001 seconds, and the control is updated at a rate of 0.01 seconds $(100 \mathrm{hz})$. And the angular acceleration for the incremental dynamics is predicted using the first-order backward difference formula. Also, discontinuous function sgn (s) in Eq (38) may cause chattering effect. To reduce chattering effect, the saturation function $\operatorname{sat}(\mathbf{s} / \Phi)$ which gradually decreases switching gain near the sliding surface is used.

$$
\operatorname{sat}\left(\frac{\mathbf{s}}{\Phi}\right)=\left\{\begin{array}{cc}
\frac{s_{j}}{\Phi}, & \left|\frac{s_{j}}{\Phi}\right|<1 \\
\operatorname{sgn}\left(\frac{s_{j}}{\Phi}\right), & \left|\frac{s_{j}}{\Phi}\right|>1
\end{array}\right\}, j=1, \cdots 3,6
$$

If not explicitly mentioned, the following control gain and weight matrices are applied to the comparative simulations.

$$
\begin{gathered}
\mathbf{Q}=\operatorname{diag}\left[\begin{array}{llllll}
0.25 & 0.25 & 0.25 & 0.25 & 0.25 & 0.25
\end{array}\right], \\
\mathbf{K}_{1}=\operatorname{diag}\left[\begin{array}{llllll}
8.8 & 8.8 & 8.8 & 8.8 & 8.8 & 8.8
\end{array}\right], \\
\mathbf{K}_{2}=\operatorname{diag}\left[\begin{array}{llllll}
2.25 & 2.25 & 2.25 & 2.25 & 2.25 & 2.25
\end{array}\right], \\
\mathbf{K}_{\mathrm{s}}=\operatorname{diag}\left[\begin{array}{llllll}
2.25 & 2.25 & 2.25 & 2.25 & 2.25 & 2.25
\end{array}\right], \\
\quad \mathbf{C}=\operatorname{diag}\left[\begin{array}{llllll}
0.5 & 0.5 & 0.5 & 0.5 & 0.5 & 0.5
\end{array}\right] \\
\mathbf{\Gamma}_{\Lambda}=\operatorname{diag}\left[\begin{array}{llllll}
0.01 & 0.01 & 0.01 & 0.01 & 0.01 & 0.01
\end{array}\right], \\
\Phi, \varepsilon=0.01 .
\end{gathered}
$$



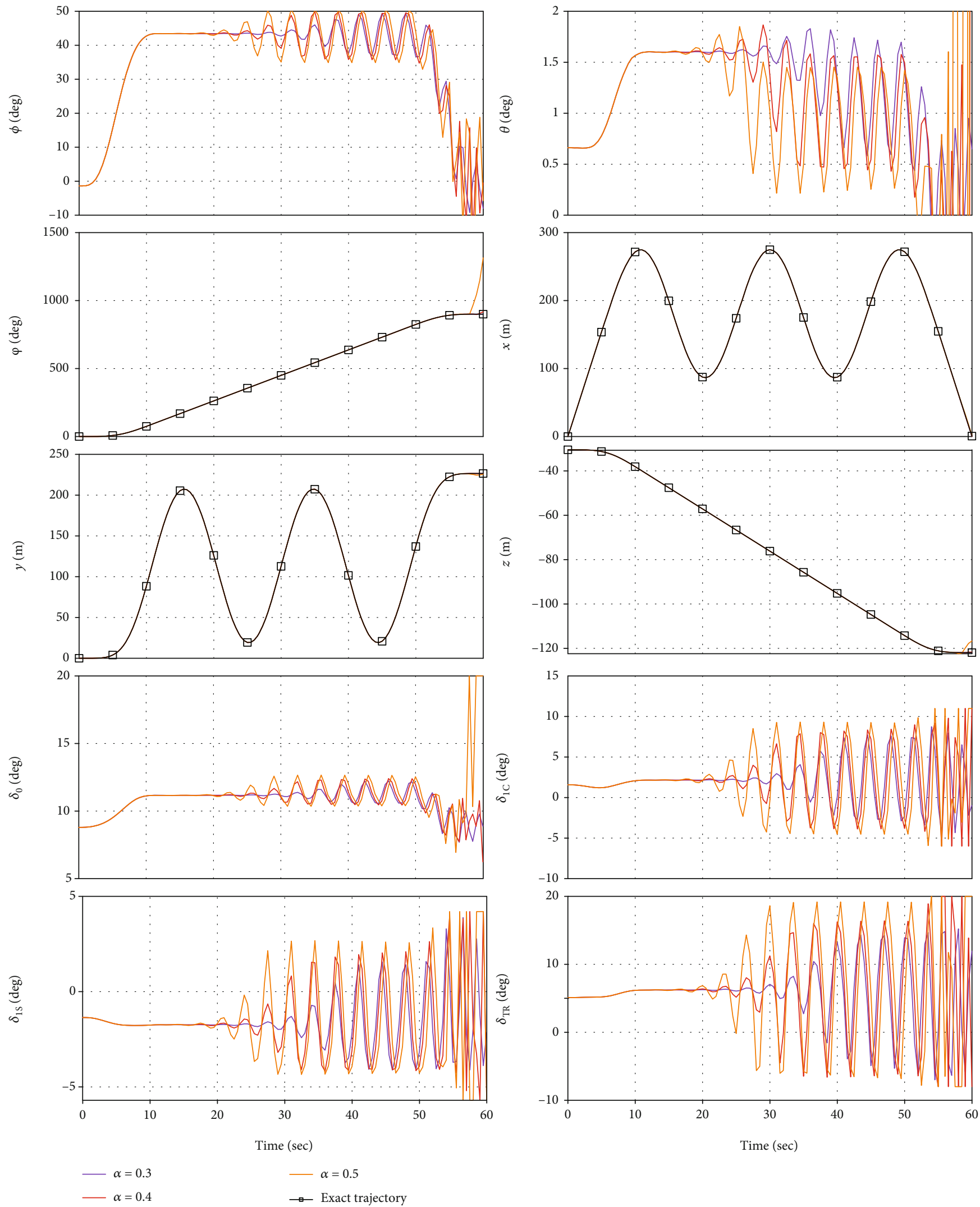

FIGURE 6: Simulated trajectories and feedback controls for the IBSC design with different levels of mismatched uncertainties (continued). 

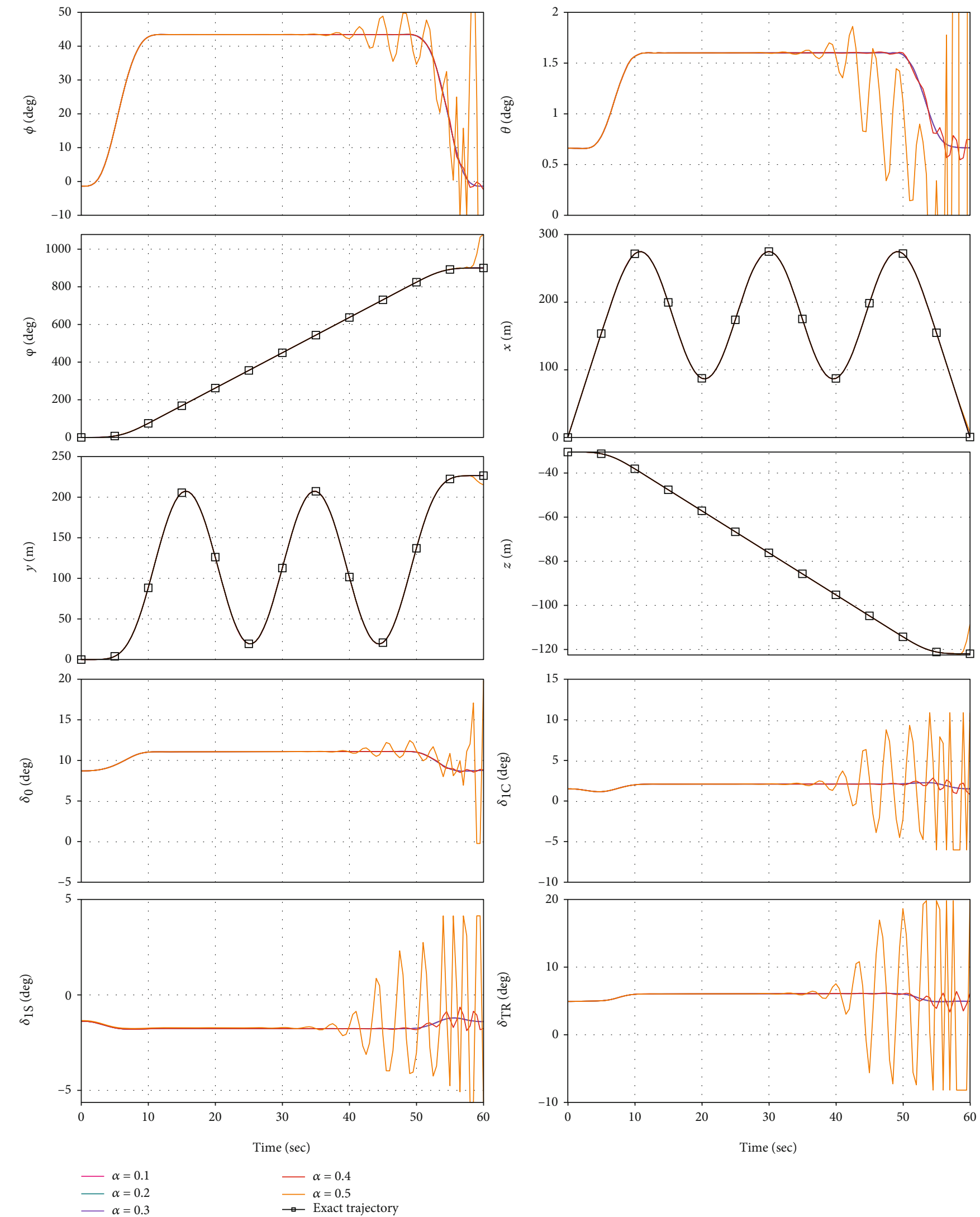

FIGURE 7: Simulated trajectories and feedback controls for the IBSC-SMC design with different levels of mismatched uncertainties with the fixed switching gain of $\Lambda_{\mathrm{sw}}=0.001$. 
The designed controllers are evaluated through their applications to the trajectory-tracking control problem for the helical-turn maneuver. This maneuver is an extended version of the transient turn MTE in ADS-33E-PRF [2] and requires large changes in the control inputs for all channels. The maneuver is started from the level flight at 60 knots and returned to the same flight condition after performing a 720 degrees heading change with the altitude increase of $300 \mathrm{ft}$ for 60 seconds. Therefore, the trajectory-tracking performance for all control channels can be evaluated through the simulation studies. The reference trajectory is generated using the seventh-order spline interpolation, with which the required position, velocity, and acceleration vectors can be straightforwardly computed for the controller implementation.

First, the IBSC design without the SMC switching element $\left(\Gamma_{\Lambda}=0\right)$ is evaluated with different levels of matched uncertainties. The simulated aircraft responses and computed feedback controls are compared in Figure 2. The prescribed position and heading trajectories are well tracked regardless of the simulated uncertainty levels from $\alpha=0$ to $\alpha=0.5$. However, it is manifested from the controls and attitude responses that the controller becomes destabilized when the sizing parameter $\alpha$ for the matched uncertainties is greater than or equal to 0.2 . At this point, it is worth mentioning that the pitch and roll attitude angles well represent their typical variations for a turning flight even if their desirable trajectories are prescribed with fixed trim values. Therefore, it can be claimed that the proposed slack variable approach coupled with the SAStype stabilization of the unstable internal attitude dynamics can greatly reduce the workloads in the present nonlinear control designs. Secondly, the performances of the IBSC-SMC design are investigated for two different fixed switchinggains of $\Lambda_{\mathrm{sW}}=0.001$ and $\boldsymbol{\Lambda}_{\mathrm{sw}}=0.01$ at the same analysis conditions as above. Even with the smaller gain of $\Lambda_{\mathrm{sw}}=0.001$, the closed-loop system becomes stable up to $\alpha=0.4$. And the trajectory-tracking performance with $\Lambda_{\mathrm{sw}}=0.01$ is maintained for all tested levels of the mismatched uncertainties as shown in Figure 3.

Finally, the simulation studies are performed with the present AIBS-SMC design with the zero initial switching gain matrices. All simulations with different levels of uncertainty show indiscernible differences in their excellent trajectorytracking performances as shown in Figure 4. The adaptive switching gains computed using Eq. (37) are presented in Figure 5. For all cases, the roll and pitch attitudes remain stable. Therefore, it can be claimed that the present AIBS-SMC design outperforms the IBSC design and IBS-SMC with the fixed switching gain in the control robustness to the matched uncertainty.

\section{Conclusions}

Typical trajectory tracking controllers stabilize the angular velocity and Euler angle first and then stabilize velocity and trajectory. This approach requires a gain scheduling strategy to stabilize the closed-loop system which leads to timeconsuming design workload. On the other hand, this paper proposes direct control approach by representing the helicopter dynamics system as a trajectory variable, allowing the control design process to be much more simplified. To apply the direct control approach, the affine form of the rotorcraft dynamics systems is needed, but high-fidelity rotorcraft is generally expressed in the nonaffine system which makes the design process more complicated. To deal with this issue, the incremental dynamic approach is adopted in this paper. With the incremental dynamic approach, the system dynamics can be represented as affine systems, and accurate predicted data can compensate for the uncertainty to increase robustness. However, such robustness from predicted data still does not compensate for the uncertainty about the control derivative matrix. This limitation is confirmed through simulation results using IBSC in Figures 2 and 6 . The robustness to this uncertainty can be improved through IBSC-SMC, which integrates ISMC and IBSC. In the design process of the IBSC-SMC, the stability of a closed-loop system can be proved mathematically using integrated control when the size of uncertainty is specified. The more robustness of IBSC-SMC is confirmed in Figures 7 and 3, and it is necessary to select an appropriate switching gain. However, selecting the switching gain is another question since the size of uncertainty is cannot be specified in the practical application. There are various techniques for measuring the size of uncertainty, but they always have an inaccuracy problem. Therefore, an adaptive method is adopted for evaluates switching gain using tracking error in this paper. Finally, the stability of the closed-loop system is proofed mathematically, and the excellent tracking performance of AIBSC-SMC is confirmed.

In this paper, three type control approaches applied incremental dynamics are described with each property, but the characteristics of incremental dynamics may be difficult to apply in the real system. Since the control derivative matrix is calculated for every time step, it may burden FCC with hard computational loads. However, the results of this paper may provide a solution to this limitation. The IBSCSMC and AIBS-SMC can preserve their tracking performance accuracy under matrix uncertainties by selecting appropriate switching gain. Thus, a scheduling process using a linearized control derivative matrix may also be applied without the installation of the complex dynamical model into FCC. However, the proposed adaptive law clearly has limitations such as an overestimation problem. The monotonically increasing switching gain may cause system instability; so', research on how to estimate appropriate switching gain is still required. If these studies were developed, it can be said that an integrated design process may be applied to the real environment.

\section{Data Availability}

The simulation program data used to support the findings of this study have not been made available to protect its intellectual property rights.

\section{Conflicts of Interest}

The authors declared that there is no conflict of interest regarding the publication of this paper. 


\section{Acknowledgments}

This work was supported by the National Research Foundation of Korea (NRF) grant funded by the Korean government (MSIT) (NRF-2020R1A2C2011955). Also, this research was supported by the Basic Science Research Program through the National Research Foundation of Korea (NRF) funded by the Ministry of Education (No. 2020R1A6A1A03046811).

\section{References}

[1] G. D. Padfield, Helicopter flight dynamics: including a treatment of tiltrotor aircraft, John Wiley \& Sons, Chichester, UK, 2018.

[2] AnonADS-33E-PRF Handling Qualities Requirements for Military Rotorcraft..

[3] G. J. Leishman, Principles of helicopter aerodynamics with CD extra, Cambridge University Press, 2006.

[4] P. Van Gils, E.-J. Van Kampen, C. C. de Visser, and Q. P. Chu, "Adaptive incremental backstepping flight control for a highperformance aircraft with uncertainties," AIAA Guidance, Navigation, and Control Conference, 2016.

[5] X. Wang, E.-J. van Kampen, Q. Chu, and P. Lu, "Incremental sliding-mode fault-tolerant flight control," Journal of Guidance, Control, and Dynamics, vol. 42, no. 2, pp. 244-259, 2019.

[6] P. Lu, E.-J. van Kampen, C. de Visser, and Q. Chu, "Aircraft fault-tolerant trajectory control using incremental nonlinear dynamic inversion," Control Engineering Practice, vol. 57, pp. 126-141, 2016.

[7] P. Van Gils, Adaptive Incremental Backstepping Flight Control Applied to an F-16 Aircraft Model, Delft University of Technology, Netherland, 2015.

[8] X. Wang, Incremental sliding mode flight control, Delft University of Technology, Netherland, 2019.

[9] V. N. Sankaranarayanan, S. Roy, and S. Baldi, "Aerial transportation of unknown payloads: adaptive path tracking for quadrotors," in 2020 IEEE/RSJ International Conference on Intelligent Robots and Systems (IROS), pp. 7710-7715, Las Vegas, NV, USA, 2020.

[10] S. Roy, S. Baldi, P. Li, and V. Narayanan, "Artificial-delay adaptive control for under-actuated Euler-Lagrange robotics," IEEE/ASME Transactions on Mechatronics, vol. 4435, no. c, pp. 1-11, 2021.

[11] S. H. Lane and R. F. Stengel, "Flight control design using nonlinear inverse dynamics," Automatica, vol. 24, no. 4, pp. 471483, 1988.

[12] S. Zhang, F. Liao, Y.-Q. Cheng, and K.-F. He, "Aircraft trajectory control with feedback linearization for general nonlinear systems," in 2020 Chinese Control And Decision Conference (CCDC), pp. 3937-3944, Hefei, China, 2020.

[13] J. D. Boskovic, L. Chen, and R. K. Mehra, "Adaptive control Design for Nonaffine Models Arising in flight control," Journal of Guidance, Control, and Dynamics, vol. 27, no. 2, pp. 209217, 2004.

[14] C. Ahn, H. Kim, and Y. Kim, "Adaptive sliding mode control for non-affine nonlinear vehicle systems," AIAA Guidance, Navigation and Control Conference and Exhibit, no. 2, pp. 1741-1757, 2007.

[15] S. Sieberling, Q. P. Chu, and J. A. Mulder, "Robust flight control using incremental nonlinear dynamic inversion and angu- lar acceleration prediction," Journal of Guidance, Control, and Dynamics, vol. 33, no. 6, pp. 1732-1742, 2010.

[16] W. van Ekeren, G. Looye, R. O. Kuchar, Q. P. Chu, and E.J. Van Kampen, "Design, implementation and flight-tests of incremental nonlinear flight control methods," in 2018 AIAA Guidance, Navigation, and Control Conference, Kissimmee, Florida, 2018.

[17] P. V. Menino Simplício, Helicopter Nonlinear Flight Control Using Incremental Nonlinear Dynamic Inversion, Delft University of Technology, Netherland, 2011.

[18] X. Wang and E.-J. Van Kampen, "Incremental backstepping sliding mode fault-tolerant flight control," in AIAA Scitech 2019 Forum, San Diego, California, 2019.

[19] P. Van Gils, "Adaptive Incremental Backstepping Flight Control," AIAA Guidance, Navigation, and Control Conference, 2015.

[20] E. R. Van Oort, L. Sonneveldt, Q. P. Chu, and J. A. Mulder, "Full-envelope modular adaptive control of a fighter aircraft using orthogonal least squares," Journal of Guidance, Control, and Dynamics, vol. 33, no. 5, pp. 1461-1472, 2010.

[21] B. Ahmed and H. R. Pota, "Flight control of a rotary wing UAV using adaptive backstepping," in 2009 IEEE International Conference on Control and Automation, pp. 17801785, Christchurch, New Zealand, 2009.

[22] A. A. H. Ali, Q. P. Chu, E. Van Kampen, and C. C. De Visser, "Exploring adaptive incremental backstepping using immersion and invariance for an F-16 aircraft," in AIAA Guidance, Navigation, and Control Conference, National Harbor, Maryland, 2014.

[23] B. Zhang and Y. Cai, "Immersion and invariance based adaptive Backstepping control for body-fixed hovering over an asteroid,” IEEE Access, vol. 7, pp. 34850-34861, 2019.

[24] C. K. Lin and M. Y. Chen, "Adaptive incremental sliding mode controller for a linear permanent magnet iron core synchronous motor and chattering reduction," in 2014 Joint 7th International Conference on Soft Computing and Intelligent Systems (SCIS) and 15th International Symposium on Advanced Intelligent Systems (ISIS),, pp. 1059-1063, Kitakyushu, Japan, 2014.

[25] S. Hussain, F. M. Malik, O. Altaf, and N. Ahmad, "High gain observer based adaptive SMC for longitudinal dynamics of a customized mini UAV," in Proceedings of the 2018 International Conference on Service Robotics Technologies-ICSRT '18 - ICSRT '18, pp. 27-31, chengdu, China, 2018.

[26] Y.-J. Huang, T.-C. Kuo, and S.-H. Chang, "Adaptive slidingmode control for nonlinearsystems with uncertain parameters," IEEE Transactions on Systems, Man, and Cybernetics, Part B (Cybernetics), vol. 38, no. 2, pp. 534-539, 2008.

[27] F. Plestan, Y. Shtessel, V. Bregeault, and A. Poznyak, "New methodologies for adaptive sliding mode control," International Journal of Control, vol. 83, no. 9, pp. 1907-1919, 2010.

[28] M. Van, M. Mavrovouniotis, and S. S. Ge, "An adaptive backstepping nonsingular fast terminal sliding mode control for robust fault tolerant control of robot manipulators," IEEE Transactions on Systems, Man, and Cybernetics: Systems, vol. 49, no. 7, pp. 1448-1458, 2019.

[29] S. Roy, S. Baldi, and L. M. Fridman, "On adaptive sliding mode control without a priori bounded uncertainty," Automatica, vol. 111, 2020.

[30] Y.-H. Yoon, C. Yang, C. Kim, and I. Joe, Flight Dynamic Analysis Program, The korean Society for Aeronauticla \& Sciences, 2012. 
[31] C.-J. Kim, K.-C. Shin, C. Yang, and I.-J. Cho, Interface Features of Flight Dynamic Analysis Program, 1st Asian Australian Rotorcraft Forum and Exhibition, 2012.

[32] D. Lee, H. Lim, H. Kim, Y. Kim, and K.-J. Seong, “Adaptive image-based visual servoing for an underactuated quadrotor system," Journal of Guidance, Control, and Dynamics, vol. 35, no. 4, pp. 1335-1353, 2012.

[33] M. Kim, Y. Hong, S. Lee, and Y. Kim, "Slack variables generation via QR decomposition for adaptive nonlinear control of affine underactuated systems," IFAC-PapersOnLine, vol. 49, no. 17, pp. 188-193, 2016.

[34] Y. Hong and Y. Kim, "Integrated design of rotary UAV guidance and control systems utilizing sliding mode control technique," International Journal of Aeronautical and Space Sciences, vol. 13, no. 1, pp. 90-98, 2012.

[35] C. J. Kim, C. Y. Yun, and S. Choi, "Fully implicit formulation and its solution for rotor dynamics by using differential algebraic equation (DAE) solver and partial periodic trimming algorithm (PPTA)," 31st European Rotorcraft Forum, vol. 2005, pp. 1-13, 2005.

[36] Chang-Joo Kim and Sangkyung Sung, "Efficient ST techniques for nonlinear optimal control analyses using a pseudospectral framework," IEEE Transactions on Control Systems Technology, vol. 23, no. 3, pp. 1110-1116, 2015.

[37] C.-J. Kim, S. H. Lee, and S. W. Hur, "Kinematically exact inverse-simulation techniques with applications to rotorcraft aggressive-maneuver analyses," International Journal of Aeronautical and Space Sciences, vol. 21, no. 3, pp. 790-805, 2020.

[38] L. Sun, C. C. de Visser, Q. P. Chu, and J. A. Mulder, "Joint sensor based backstepping for fault-tolerant flight control," Journal of Guidance, Control, and Dynamics, vol. 38, no. 1, pp. 62-75, 2015.

[39] T. Madani and A. Benallegue, "Backstepping control for a quadrotor helicopter," in 2006 IEEE/RSJ International Conference on Intelligent Robots and Systems, pp. 3255-3260, Beijing, China, 2006.

[40] Z. Zheng and H. Yi, "Backstepping control design for UAV formation with input saturation constraint and model uncertainty," in 2017 36th Chinese Control Conference (CCC), pp. 6056-6060, Dalian, China, 2017.

[41] Z. Song and K. Sun, “Adaptive backstepping sliding mode control with fuzzy monitoring strategy for a kind of mechanical system," ISA Transactions, vol. 53, no. 1, pp. 125-133, 2014.

[42] F. J. Lin, P. H. Shen, and S. P. Hsu, "Adaptive backstepping sliding mode control for linear induction motor drive," IEE Proceedings: Electric Power Applications, vol. 149, no. 3, pp. 184-194, 2002. 